University of Wollongong

Research Online

Faculty of Engineering and Information

Faculty of Engineering and Information

Sciences - Papers: Part A

Sciences

$1-1-2014$

Online voltage control in distribution systems with multiple voltage regulating devices

Dothinka Ranamuka Rallage

University of Wollongong, dssrr987@uowmail.edu.au

Ashish Agalgaonkar

University of Wollongong, ashish@uow.edu.au

Kashem Muttaqi

University of Wollongong, kashem@uow.edu.au

Follow this and additional works at: https://ro.uow.edu.au/eispapers

Part of the Engineering Commons, and the Science and Technology Studies Commons

Research Online is the open access institutional repository for the University of Wollongong. For further information contact the UOW Library: research-pubs@uow.edu.au 


\title{
Online voltage control in distribution systems with multiple voltage regulating devices
}

\begin{abstract}
Voltage regulation in distribution systems is typically performed with the aid of multiple voltage regulating devices, such as on-load tap changer and step voltage regulators. These devices are conventionally tuned and locally coordinated using Volt/VAR optimization strategies in accordance with the time-graded operation. However, in case of distribution systems with distributed generation (DG), there could be a possibility of simultaneous responses of DG and multiple voltage regulators for correcting the target bus voltage, thereby resulting in operational conflicts. This paper proposes an online voltage control strategy for a realistic distribution system containing a synchronous machine-based renewable DG unit and other voltage regulating devices. The proposed strategy minimizes the operational conflicts by prioritizing the operations of different regulating devices while maximizing the voltage regulation support by the DG. It is tested on an interconnected medium voltage distribution system, present in New South Wales, Australia, through time-domain simulation studies. The results have demonstrated that voltage control for a distribution feeder can effectively be achieved on a real-time basis through the application of the proposed control strategy.
\end{abstract}

\section{Keywords}

distribution, devices, regulating, multiple, systems, control, voltage, online

\section{Disciplines}

Engineering | Science and Technology Studies

\section{Publication Details}

D. Ranamuka, A. P. Agalgaonkar \& K. M. Muttaqi, "Online voltage control in distribution systems with multiple voltage regulating devices," IEEE Transactions on Sustainable Energy, vol. 5, (2) pp. 617-628, 2014. 


\title{
On-line Voltage Control in Distribution Systems with Multiple Voltage Regulating Devices
}

\author{
D. Ranamuka, A. P. Agalgaonkar, Member, IEEE, and K. M. Muttaqi, Senior Member, IEEE
}

\begin{abstract}
Voltage regulation in distribution systems is typically performed with the aid of multiple voltage regulating devices, such as on-load tap changer (OLTC) and step voltage regulators (SVRs). These devices are conventionally tuned and locally coordinated using Volt/VAR optimization strategies in accordance with the time graded operation. However, in case of distribution systems with distributed generation (DG), there could be a possibility of simultaneous responses of DG and multiple voltage regulators for correcting the target bus voltage, thereby resulting into operational conflicts. This paper proposes an on-line voltage control strategy for a realistic distribution system containing synchronous machine based renewable DG unit and other voltage regulating devices. The proposed strategy minimizes the operational conflicts by prioritizing the operations of different regulating devices while maximizing the voltage regulation support by the DG. It is tested on an interconnected medium voltage distribution system, derived from the state of New South Wales, Australia, through time domain simulation studies. The results have demonstrated that voltage control for a distribution feeder can effectively be achieved on real-time basis through the application of the proposed control strategy.
\end{abstract}

Index Terms--distribution system; distributed generation; onload tap changer; step voltage regulator; voltage regulation.

\section{INTRODUCTION}

$\mathrm{T}_{\mathrm{v}}$ HE conventional voltage regulation by means of multiple voltage regulating devices such as on-load tap changer (OLTC) and/or step voltage regulators (SVRs) may need to be updated in the presence of distributed generation (DG) units, which are conventionally operated at unity power factor to comply the requirement of no active regulation of the voltage at the point of common coupling as detailed in IEEE standard $1547^{\mathrm{TM}}$ - 2003: Standard for Interconnecting Distributed Resources with Electric Power Systems. Since there is no realtime control to avoid simultaneous responses of multiple voltage regulators and DG, there could be conflicting operations under certain system operating conditions. This may cause increased number of tap operations resulting into voltage variations. Also, the changes in active and reactive power flows between SVR and load centre, due to the active and reactive power injection by the DG, may affect the line drop compensation (LDC) scheme of the SVR. Authors of [1] and [2] have investigated the voltage and reactive power

The authors are with the Australian Power Quality and Reliability Centre, School of Electrical, Computer and Telecommunications Engineering, University of Wollongong, New South Wales 2522 Australia. (e-mail: dssrr987@uowmail.edu.au; ashish@uow.edu.au; kashem@uow.edu.au) control issues in a distribution system with substation OLTC, capacitor banks and DG. In [3], interactions between DG and SVR have been investigated. The voltage control issues in case of a realistic distribution network with substation OLTC and multiple distributed generators have been investigated in [4]. Authors of [5] have investigated the operation of multiple voltage regulators and synchronous machine based DG unit in case of realistic medium voltage (MV) distribution feeder using time domain simulation studies.

The synchronous machine based distributed generators can effectively be utilized for the voltage regulation process in the distribution networks within their active and reactive power capability limits [6]. A control strategy has been proposed in [7] which can appropriately select the optimal operating points with requisite active and reactive power injections for ensuring maximum voltage support by the DG. In [8], a voltage control strategy is proposed for DG using the voltage control mode and the power factor control mode. In [9], the proportional and integral type controller is used for voltage control by DG, and also an advanced LDC scheme is proposed in order to regulate the voltage at a remote node by the DG. In [10], a local, intelligent and auto-adaptive voltage regulator for DG is proposed. A fuzzy based digital automatic voltage regulator is proposed in [11] for small scale synchronous generators with unbalanced loads. However, these are all knowledge based controls. An optimization technique is proposed in [12] for coordinated voltage control in distribution networks using DG. In [13], an adaptive self-tuning automatic voltage regulator is designed for updating the reference voltage for the excitation system of synchronous machine based DG unit.

In case of MV distribution systems, especially for long distribution feeders with DG, there could be significant amount of conflicting operations between multiple voltage regulating devices and DG. This paper proposes an on-line voltage control strategy for correcting the feeder voltage with appropriate control-coordination ascertaining voltage regulation support by synchronous machine based renewable DG unit. It ensures prioritized operation of the DG unit in conjunction with the voltage regulating devices, and aids in blocking simultaneous operations between the DG unit and voltage regulating devices; thereby minimizing the total tap operations. Since the proposed control logic preserves the existing design philosophies of DG local voltage controllers and step voltage regulators, and exhibits high computational efficiency, it can be easily implemented on a practical distribution system via a substation centered distribution 
management system (DMS). The proposed on-line control strategy has been tested on a realistic MV distribution network using MATLAB-SimPowerSystems and results are presented.

The paper is organized as below. Section II discusses the operation of multiple voltage regulating devices and DG; Section III conceptualizes on-line voltage control; Section IV outlines proposed control strategy; Section V elaborates realistic case study using time domain simulations while Section VI concludes the paper.

\section{Operation of Multiple Voltage Regulating DeVices AND DG IN A DISTRIBUTION SYSTEM}

In case of long MV distribution feeders, there could be significant drop in voltage towards the end of the feeder, especially due to the voltage drop across the line sections attributed by random variation in the load demands. It is essential to adopt necessary measures to maintain the voltage within stipulated limits throughout the feeder. Accordingly, multiple step voltage regulators are normally used in conjunction with the OLTC at the substation to regulate the voltage for long distribution feeders. A topology of typical distribution system with multiple step voltage regulators (i.e. SVR1, SVR2 and SVR3), OLTC and DG is shown in Fig. 1. If DG is operated in an uncoordinated fashion, there is a possibility to introduce strong control interaction with other regulating devices in the network, due to simultaneous response of DG and other regulating devices to correct the network voltage [5].

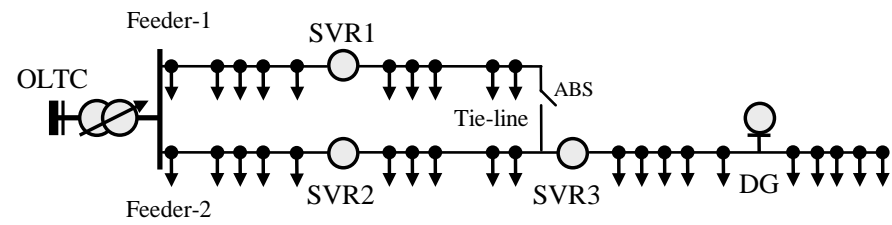

Fig. 1. A topology of distribution system with multiple voltage regulating devices and DG.

\section{A. Substation On-Load Tap Changer and Step Voltage Regulators}

Substation OLTC serves the core voltage regulation in distribution feeders fed by the substation. SVRs are used for voltage regulation, especially for regulating the voltage further at the downstream of a distribution feeder. Most of the voltage regulators consist of an auto-transformer equipped with a load tap changing mechanism. The voltage change is obtained by changing taps on the series winding of the auto-transformer. SVRs are classified into several types according to their construction and mode of operation [14]. Typically, SVRs can be configured using a Type A or Type B connection. The more common type is Type B connection, where the input to the SVR is across the series and shunt windings. Therefore, when a change in taps is required, the autotransformer changes the number of turns on the series winding. The function of substation OLTC and SVR, with the application of an analog or digital LDC circuit, for estimating the voltage can generally be explained with the aid of voltage regulating device controller. It keeps the target load centre voltage $V_{L C}$ within a specified range. The set (reference) voltage, $V_{S E T / V R}$ and deadband voltage, $V_{D B}$, as shown in (1), are the controller parameters for the voltage regulating device where $V_{L B}$ and $V_{U B}$ are the lower boundary and upper boundary limits, respectively.

$$
\begin{aligned}
& V_{L B} \leq V_{L C} \leq V_{U B} \\
& V_{L B}=V_{S E T / V R}-0.5 V_{D B}, V_{U B}=V_{S E T / V R}+0.5 V_{D B}
\end{aligned}
$$

The function of a LDC based SVR in forward mode can be explained using the expression given by (2). It is assumed that $R_{\mathrm{L}}$ and $X_{\mathrm{L}}$ are the set values of resistance and reactance for LDC circuit respectively, and $I$ is the measured current at the load side of SVR. It is assumed that the voltage at the voltage regulating device connection point is $V_{V R}$. For a lagging power factor $\cos (\varphi)$, the remote end voltage can be given by (2).

$$
V_{L C} \approx V_{V R}-I \times R_{L} \times \cos (\varphi)-I \times X_{L} \times \sin (\varphi)
$$

The changes in active and reactive power flows between voltage regulating device and load centre will affect the performance of a LDC based SVR. The performance will also be deteriorated if the $X_{L} / R_{L}$ ratio of LDC setting is poorly adjusted. This LDC error may cause additional tap operations of the SVR.

On the other hand, the effect of a voltage regulating device on its downstream nodal voltage profile can be estimated using the lossless linear regulator model given by (3), where $V_{L C(j)}{ }^{u r}$ is the unregulated voltage at its target load centre, $V_{i}{ }^{u r^{\prime}}$ and $V_{i}^{r s}$ are the voltages at any $i^{\text {th }}$ node before and after the voltage control action respectively, and $T_{j}^{r}$ is the equivalent tap ratio of $j^{\text {th }}$ device for regulating the load centre voltage defined by the voltage set value $V_{\text {SETVR(j) }}$ [15].

$$
T_{j}^{r}=\frac{V_{S E T / V R(j)}}{V_{L C(j)} r^{\prime}}, \quad V_{i}^{r S}=T_{j}^{r} \times V_{i}^{u r^{\prime}}
$$

The voltage control parameters of a substation OLTC i.e. set voltage and dead band are typically set to regulate the secondary voltage without violating the stipulated limits. The substation OLTC time delay can be set based on constant time variant or inverse time characteristics. In case of constant time variant characteristics, the time delay is constant. With inverse time characteristics, the time delay is inversely proportional to the voltage deviation and can dynamically be updated according to the network conditions. The set voltages and dead band settings of the SVRs are selected based on the total number of taps and the percentage value of voltage regulating range respectively. They are operated with a time delay. SVR farther from the substation has longer time delay than the SVR closer to the substation [16]. This time delayed operation of SVRs is based on constant time variant characteristics. Non sequential or sequential operational strategies are used for SVR tap operations and to locally coordinate their operations with the upstream regulators. In non-sequential operation, the 
time delay is used for all the tap steps, while in sequential operation the time delay is used only for the first tap operation and the subsequent taps are operated with mechanical time delay.

\section{B. Effect of DG on the Nodal Voltage Profile of a Feeder}

Since, both active and reactive power injections from DG can change the distribution feeder voltage profile; impedance matrix (Z-matrix) of the distribution feeder can generally be used for estimating the respective voltage change [5]. If the DG current is $I_{g}$, then the voltage change by DG at $k^{\text {th }}$ nodal bus, $V_{k / D G}$ can be estimated by using (4). It is assumed that the distribution feeder has $n$ number of nodal buses (i.e. $k=1, \ldots$, n) and DG is connected at $g^{\text {th }}$ node.

$$
\begin{aligned}
& {[\Delta V]_{n \times 1}=[Z]_{n \times n} \times[\Delta I]_{n \times 1}} \\
& \text { where } V_{k / D G}=Z_{k, g} \times I_{g}
\end{aligned}
$$

The impact of DG on feeder voltage can be significant if the DG is connected at the end of a lightly loaded long distribution feeder [5]. Synchronous machine based DG units are capable of responding instantaneously to meet the system load demand.

\section{Effect of simultaneous Responses of Voltage Regulating Devices and DG on Nodal Voltage Profile of a Feeder}

Based on the inherent operational characteristics of synchronous machine based DG and SVRs, there is a potential of simultaneous response between voltage regulating devices and DG under conventional voltage control. If there are tap operations for multiple SVRs and a DG response at the same time then their cumulative effect on the voltage at $i^{\text {th }}$ node, $V_{i}$ can be estimated using (5). In this paper, it is assumed that the substation OLTC is fixed, and DG is operated in power factor control mode for conventional operation. The total voltage change by simultaneous tap operations is $\Sigma V_{i}^{r s}$ and the voltage change by $\mathrm{DG}$ is $V_{\mathrm{i} / \mathrm{DG}}$.

$$
V_{i}=\sum V_{i}^{r s}+V_{i / D G}
$$

In the worst case scenario, $V_{i}$ can be exceeded beyond $V_{\max }$ (maximum allowable voltage limit for the distribution system) thereby violating the dead-band values of voltage regulating devices. Hence, the simultaneous response of voltage regulating devices and DG can lead to conflicting operations under certain system operating conditions and may exhaust total number of tap operations resulting into voltage variations.

\section{PRACTICAL IMPLEMENTATION STRATEGy FOR PROPOSED ON-LiNE VOLTAGE CONTROL}

The proposed control strategy described in Section IV can be implemented with the aid of a DMS. It mainly relies on three components, which are as follows.

(a) Control module for maximizing voltage regulation support by synchronous machine based DG unit. (b) Capability to capture on-line measurements of load center voltage for SVR and DG voltage control module operation.

(c) Control module for blocking simultaneous operations of DG voltage control module and multiple SVR taps.

For implementing the proposed control system, the technical considerations and features related to commercially available DMS [17], [18] have been explored. A DMS is a real-time information system for all operational activities in a modern distribution control center. The advanced DMS continually runs real-time analysis for various distribution system functionalities such as service restoration, Volt/VAR control, adaptable Volt/VAR optimization, and equipment condition monitoring in addition to the state estimation. The state estimation algorithms applied for MV distribution systems may use network characteristics and data, estimated load and the power output by DG (pseudo-measurements). State estimation outputs are the controller inputs. A significant reduction in number of remote measurements can be obtained through optimal siting of measurement nodes. The substation centered advanced DMS schemes are well suited for waveform analysis and the signal processing, including relevant real-time monitoring and control [18]. Also, these DMS schemes are capable of utilizing user-defined algorithms and customized software to determine best operating settings for voltage control devices such as DGs and tap changers in real-time [19].

The control system proposed in this paper is based on updating the reference voltage of DG excitation control and blocking the simultaneous operations between DG voltage control module and SVR taps in real-time; in addition to tuning the voltage controller parameters. The proposed control modules can be easily implemented in practical distribution systems containing multiple SVRs and DG. Accordingly, the newly designed control modules can be integrated into a substation centered DMS for enhancing real-time control operation. A substation centered DMS is proposed in this paper for enacting the proposed control strategies. The topology of the proposed system implementation is shown in Fig. 2.

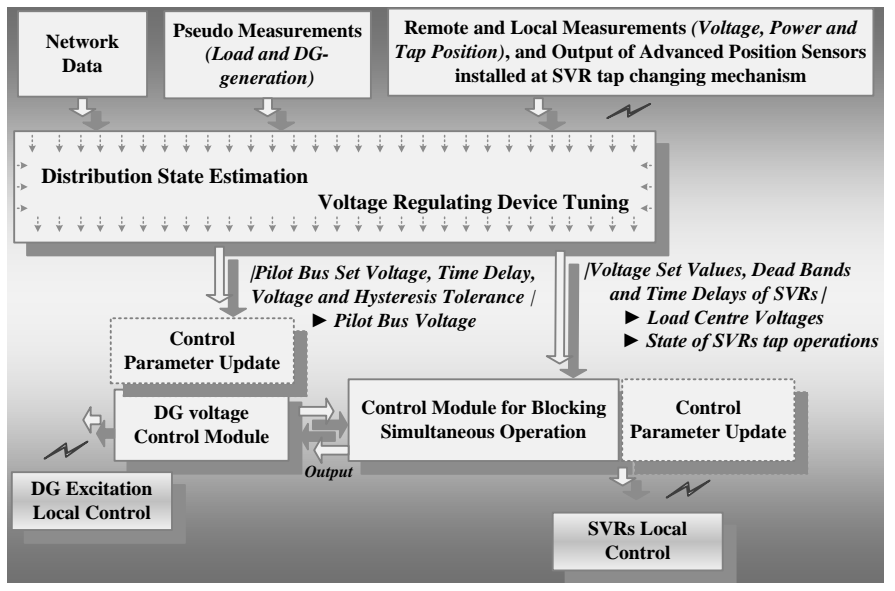

Fig. 2. Topology of the on-line implementation for proposed control strategy. 
Multiple voltage regulating devices and DG voltage controller parameters are tuned and updated on daily basis to ensure reliable voltage control. The control settings of DG voltage control module and, control settings of SVR and substation OLTC local controllers are tuned by a tuning algorithm, which can be embedded in the DMS. The time delay sequence of multiple voltage regulating devices in a feeder should be designed based on the condition given by (6), where SVRs are counted from the substation end.

$$
T_{D G}<<T_{S V R(1)}<T_{S V R(2)}<\ldots T_{S V R(n)}<T_{O L T C}
$$

In (6), the $T_{D G}$ and $T_{S V R}$ are time delays for DG voltage control module and the SVRs respectively, which are assigned with constant time variant characteristics for non-sequential operation. The DG voltage control module (DG-VCM) and control module for blocking simultaneous operations (CMBSO) are integrated in the DMS as separate controls. The DGVCM output is an input to the CM-BSO, where its output referred to the operation of DG-VCM is sent to the DG-VCM as an input. The respective control parameters are updated using separate modules as shown in Fig. 2. Additional twoway communication channels may be required for updating the control settings of DG excitation controller and SVRs.

The proposed voltage control strategy enhances the system security due to its capability to update settings and enforce quick changeover to local control. It also helps to implement the proposed control scheme with minimum modification in the conventional control scheme as follows.

(a) DMS-tap changer control interface for updating control settings and assigning on-line target point voltage, and incorporating decision variables of CM-BSO.

(b) Change-over facility to enable local control whenever required.

(c) Advanced position sensors at the tap changing mechanism including associated interface for determining the state of progressive tap operation.

(d) DMS-DG excitation control interface for updating reference voltage of the excitation control, and incorporating decision variables of CM-BSO.

\section{Proposed Control Strategy}

The proposed control strategy is based on the following three main control aspects managed by DMS in addition to tuning the regulating devices and prioritizing their operations: (a) Maximizing voltage regulation support by DG through DGVCM, (b) On-line load center voltage measurements for SVR operation, and (c) Real-time control for avoiding simultaneous operation of DG-VCM and multiple SVR taps.

In case of the control aspect (a), the DG operates as a voltage regulating device with its fast excitation control action. Accordingly, the associated time delay is smaller than the mechanical time delay of a SVR [1]. Also, the reference voltage for the excitation system is appropriately updated, by maintaining the time delay sequence given by (6), for maximizing the voltage regulation support by DG through on- line DG-VCM. Therefore, the voltage change by means of a DG response, $V_{i / D G}$ as given in (5), is now a control variable (defined as $V_{i / D G C}$ ), and the system voltage is quickly regulated by the DG under all system conditions. On the other hand, in case of the aspect (c), the simultaneous operations of SVR taps and DG-VCM are blocked in real-time preserving their local control action for which the $V_{i}$ can be expressed by (7).

$$
V_{i}=V_{i}^{r s}+V_{i / D G C}
$$

Since, one of the terms in (7) is appropriately controllable; any violation in the system voltage, given by (5), can be avoided by the proposed on-line voltage control strategy, while maximizing and prioritizing the voltage regulation support by the DG. It also aids distribution system by enabling one voltage control action (of DG-VCM or SVR tap) at a time.

\section{A. DG Voltage Control Module (DG-VCM)}

The voltage regulation by DG can be more significant in case of lightly loaded long distribution feeders [5]. The pilot bus (target bus) voltage is monitored in real-time, where pilot bus can be the weakest bus (or remote bus in case of radial distribution feeder). In accordance with the magnitude of pilot bus voltage, reference voltage for the excitation system of DG, $V_{\text {ref }}$ will be updated by exploiting the reactive capability of the machine; thereby aiding voltage regulation. The lower and upper limits of reference voltage can be derived based on the capability chart of the synchronous machine in order to maximizing the DG voltage regulation support.

The selection of pilot bus and tuning of control parameters need to be carried out based on the DG capacity and standard voltage limits for the network. It is assumed that the initial exciter setting for the DG is at the nominal value of reference voltage. Subsequently, the proposed DG voltage control module (DG-VCM) embedded in the DMS is enabled. Its topology is shown in Fig. 3.

The voltage set value $V_{S E T / P}$, voltage tolerance value $V_{t}$ and hysteresis tolerance value $\varepsilon_{t}$ are included in the DG-VCM control layer-1 which determines the state of the voltage error $V_{e r r}$. The difference between magnitude of pilot bus voltage $V_{p}$ and the respective $V_{S E T / P}$ is the voltage error $V_{\text {err }}$. The time delay $T_{D G}$ is assigned in the DG-VCM control layer-2 which determines the time duration (T) of $V_{\text {err }}$ exceeding the $V_{t}$. If the time duration $T$ exceeds the assigned time delay value $T_{D G}$, the decision making layer (DG-VCM control layer-3) is enacted. The inputs of DG-VCM control layer-2 and DG-VCM control layer-3 are $V_{m e}$ and $V_{d}$, respectively. Accordingly, the DG excitation control scheme is updated with a new reference voltage by a voltage change $(a)$ per control action. It is noted that the voltage at certain buses can be regulated with the use of proposed control scheme, especially in the presence of synchronous machine based DG. The representative control logic for the model can be summarized as given by (8) to (12). 


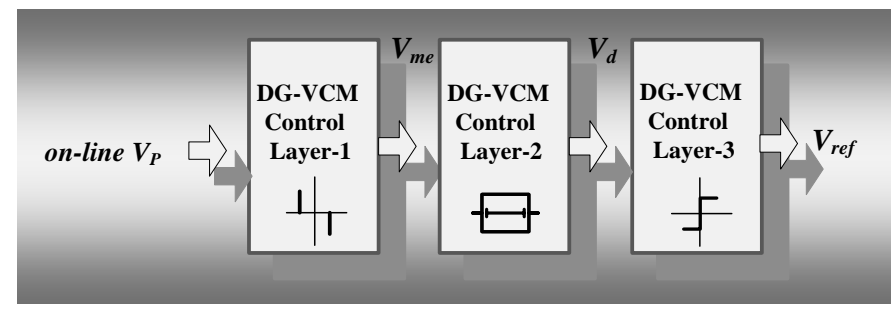

Fig. 3. Topology of the DG voltage control module.

$V_{\text {err }}=V_{S E T / P}-V_{P}$

$V_{m e}= \begin{cases}+1 & \text { for } V_{\text {err }}>\left(V_{t}+\epsilon_{t}\right) \\ & \text { for } V_{t}<V_{\text {err }} \leq\left(V_{t}+\epsilon_{t}\right), V_{\text {err }} \downarrow \\ 0 & \text { for }-V_{t} \leq V_{\text {err }} \leq+V_{t} \\ & \text { for } V_{t}<V_{\text {err }} \leq\left(V_{t}+\epsilon_{t}\right), V_{\text {err }} \uparrow \\ & \text { for }-\left(V_{t}+\epsilon_{t}\right) \leq V_{\text {err }}<-V_{t}, V_{\text {err }} \downarrow \\ -1 & \text { for } V_{\text {err }}<-\left(V_{t}+\epsilon_{t}\right) \\ & \text { for }-\left(V_{t}+\epsilon_{t}\right) \leq V_{\text {err }}<-V_{t}, V_{\text {err }} \uparrow\end{cases}$

$T= \begin{cases}T=(T+\Delta T) & \text { if } V_{\text {me }} \neq 0 \\ 0 & \text { if } V_{\text {me }}=0 \\ & \text { if } V_{\text {me }} \pm \overrightarrow{(F}^{\mp} \\ & \text { if } V_{\text {ref }} \rightarrow\left(V_{\text {ref }}\right)_{\text {new }} \text { transition state }\end{cases}$

$V_{d}= \begin{cases}V_{m e} & \text { if } T>T_{D G} \\ 0 & \text { if } T \leq T_{D G}\end{cases}$

$V_{\text {ref }}= \begin{cases}V_{\text {ref }} & \text { if } V_{d}=0 \\ V_{\text {ref }}+a & \text { if } V_{d}=+1 \\ V_{\text {ref }}-a & \text { if } V_{d}=-1\end{cases}$

The time delay, $T_{D G}$ has been introduced to prevent unnecessary control actions in case of transient voltage variations. It is set to a small value with constant time variant characteristics in order to prioritize the voltage regulation support by DG as given in (6). Also, the values of $a$ and $V_{t}$ should be adequately large for stable operation.

\section{B. On-Line Voltage Measurements for Step Voltage Regulator Operation}

The changes in active and reactive power flows between SVR and load centre may lead to a higher LDC error resulting into increased number of tap operations. If nodal voltage at the load centre is measured on-line, the conventional LDC scheme can be disabled. This paper proposes a real-time monitoring scheme for SVRs. The topology of control model of such a SVR is shown in Fig. 4.

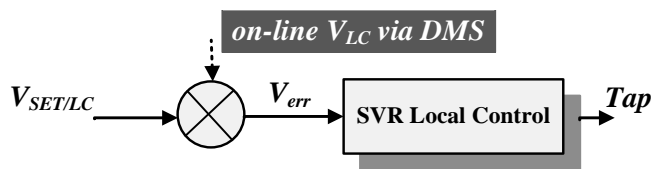

Fig. 4. Topology of the model for SVR control with on-line load centre voltage measurements.

\section{Control Module for Blocking Simultaneous Operation (CM-BSO)}

A control module is proposed for blocking simultaneous operations of DG-VCM and multiple SVR taps in real-time, which also ensures the prioritized action of the respective controllers. Since substation OLTC deals with voltage regulation at the substation bus-bar level using a coarse control and operates after a prolonged time delay, it is not incorporated in the fine control strategy adopted in this control module. The topology of the proposed control module (CMBSO) embedded in the DMS is shown in Fig. 5.

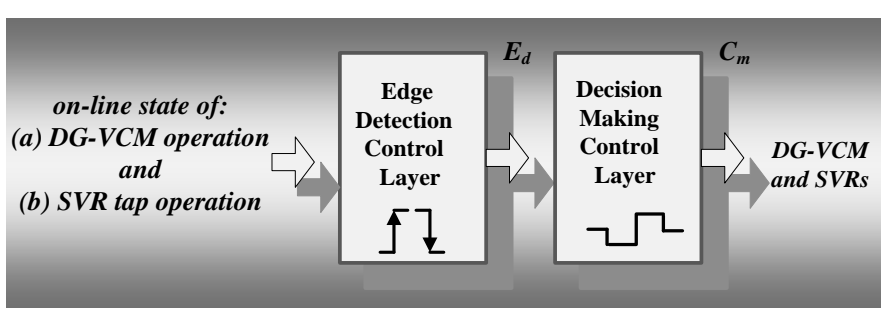

Fig. 5. Topology of the control module for blocking simultaneous operations.

The inputs for edge detection control layer are the state of DG-VCM operations and the tap operations of multiple SVRs. The position sensors can monitor transition state of a tap i.e. up or down operation with rising or falling edge at their output, respectively. Transition state of a DG-VCM operation can directly be monitored as rising or falling edge from its output. The edge detection control layer detects the state of progression for DG-VCM operations and each SVR tap operation by separate edge detection modules in order to enact the decision making control layer. Accordingly, rising or falling edge is referred to a transition state of a progressive tap operation or a DG-VCM operation. The 2D step edge detection model, proposed in [20], is adopted in the proposed control module.

The decision making control layer is used for processing the outputs of edge detection control layer by summing up modules and comparator modules. The output of each summing up module is compared with the reference, assigned in a single input-inverting output comparator module prior to initiation of a control action for the DG-VCM or a tap operation for the respective SVR. The DG-VCM or the associated SVR tap operation will be enacted based on the outcome of the decision making control layer.

The representative control logic of CM-BSO for interpreting a DG-VCM operation can be summarized as given in (13) to (15). The output of the respective module for summing up the outputs of DG-VCM and SVRs edge detection modules is $F_{r}(D G-V C M)$, and the output of the comparator module is $C_{m}(D G-V C M)$. The respective modules for SVR are respectively $F_{r}(S V R)$ and $C_{m}(S V R)$. 
Edge detection module (DG-VCM / SVR)

$E_{d}(D G-V C M / S V R)=\left\{\begin{array}{cc}+1 & \text { if rising or falling edge } \\ & \text { is detected } \\ & \forall \text { transition state of a } \\ & D G-V C M / S V R \text { operation } \\ 0 & \text { otherwise }\end{array}\right.$

Summing up module ( $D G-V C M$ / SVR)

$F_{r}(D G-V C M / S V R)=\left\{\begin{array}{ccc}+1 & \text { if output of an } E_{d}(S V R) \text { and } / \\ & \text { or } E_{d}(D G-V C M) \text { is }+1 \\ & \forall \sum E_{d}(S V R)+ \\ 0 & \text { otherwise } & E_{d}(D G-V C M)\end{array}\right.$

Comparator module (DG -VCM / SVR)

$C_{m}(D G-V C M / S V R)=\left\{\begin{array}{cc}+1 & \text { if output of } F_{r}(D G-V C M) / \\ & F_{r}(S V R) \text { is } 0 \\ \quad \forall \text { Inverting output rule } \\ 0 & \text { otherwise }\end{array}\right.$

The diagrammatic representation of the proposed scheme in DG-VCM side for enacting a control action is shown in Fig.6. The representative control logic for the MOD- 1 module is given by (16).



Fig. 6. Modification required in the proposed DG-VCM.

$M O D-1$ module
$V_{m}=\left\{\begin{array}{l}V_{m e} \quad \text { if } V_{m e} \neq 0 \text { and } C_{m}(D G-V C M)=+1 \\ V_{m e} \\ \text { hold the action of } D G-V C M \text { layer }-2 \text { after the time delay } \\ \text { until } C_{m}(D G-V C M)=+1 \text {, and then enact the } \\ D G-V C M \text { layer }-3 \\ \quad \text { if } V_{m e} \neq 0 \text { and } C_{m}(D G-V C M)=0 \\ 0 \quad \text { if } V_{m e}=0 \text { and } C_{m}(D G-V C M)=0 \text { or }+1\end{array}\right.$

The diagrammatic representation of the proposed control scheme for enacting a tap operation of SVR is shown in Fig. 7. It is established with the aid of voltage regulator model proposed in [21]. The representative control logic for the MOD-2 module is given by (17).

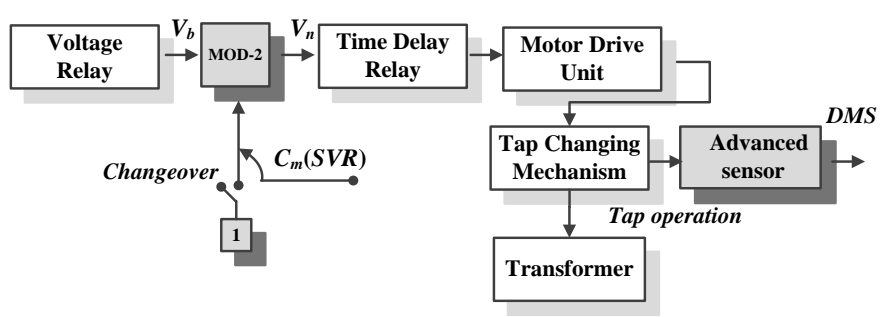

Fig. 7. Modifications required in SVR control.

$$
\begin{aligned}
& \text { MOD - } 2 \text { module } \\
& V_{n}=\left\{\begin{array}{l}
V_{b} \quad \text { if } V_{b} \neq 0 \text { and } C_{m}(S V R)=+1 \\
V_{b} \\
\text { hold the action of time delay relay after the time delay } \\
\text { until } C_{m}(S V R)=+1 \text {, and then enact the SVR tap } \\
\text { if } V_{b} \neq 0 \text { and } C_{m}(S V R)=0 \\
0 \quad \text { if } V_{b}=0 \text { and } C_{m}(S V R)=0 \text { or }+1
\end{array}\right.
\end{aligned}
$$

According to (16) and (17), no DG voltage control module and SVR tap operation can be initiated simultaneously. Hence, this scheme is capable of blocking any simultaneous operation in real-time, as it allows only one voltage control action (of DG-VCM or SVR tap) at a time, and accordingly enacts the next control action. The control algorithm of CM-BSO can be summarized as follows for the sample feeder- 2 as depicted in Fig. 1:

Step - 1 From on-line measurements and sensor information sent by DMS, the proposed and local voltage controllers are executed.

Step - 2 (i) DG-VCM will update the reference voltage, $V_{\text {ref }}$ of DG excitation control after time delay, $T_{D G}$ if the pilot bus voltage magnitude is not within the stipulated limits and there is a confirmation from the CM-BSO.

or

(ii) SVR2 will operate a tap after time delay, $T_{\text {SVR(2) }}$ if the target voltage magnitude is not within the stipulated limits and there is a confirmation from the CM-BSO.

or

(iii) SVR3 will operate a tap after time delay, $T_{\text {SVR(3) }}$ if the target voltage magnitude is not within the stipulated limits and there is a confirmation from the CM-BSO.

or

(iv) DG-VCM and SVR2 will be enabled after the associated time delays i.e. $T_{D G}<<T_{S V R(2)}$, if the target voltage magnitudes are not within the stipulated limits and there is a confirmation from the CM-BSO.

or

(v) DG-VCM and SVR3 will be enabled after the associated time delays i.e. $T_{D G}<<T_{S V R(3)}$, if the target voltage magnitudes are not within the stipulated limits and there is a confirmation from the CM-BSO. 
(vi) SVR2 and SVR3 taps will be operated after the associated time delays i.e. $T_{S V R(2)}<T_{S V R(3)}$, if the target voltage magnitudes are not within the stipulated limits and there is a confirmation from the CM-BSO.

or

(vii) DG-VCM, SVR2 and SVR3 will be operated after the associated time delays i.e. $T_{D G}<<T_{S V R(2)}<T_{S V R(3)}$, if their target voltage magnitudes are not within the stipulated limits and there is a confirmation from the CM-BSO.

Step - 3 For the subsequent instances of time (i.e. $t=t+1$ ), repeat the procedure from Step - 1.

The above procedure can be adjusted for a given network with a given number of SVRs and a DG system. If there are multiple DG units distributed to a larger extent in the MV distribution system then the proposed method can be easily extended by establishing the relevant control logic to fully utilize the voltage support capabilities of these DG units and ensure coordinated operation of all the voltage regulating devices including multiple DG units on the feeder.

\section{CASE STUDY}

In this section, simulation results and discussion associated with the proposed on-line voltage control in a realistic MV distribution system are presented.

\section{A. Test Distribution System Model}

The performance of the proposed control algorithm is demonstrated through modeling the topology of a realistic 11 $\mathrm{kV}$ distribution system in MATLAB-SimPowerSystems and conducting time domain simulations for different operational strategies of the feeders. The topology of $11 \mathrm{kV}$ test distribution network, derived from the State of New South Wales (NSW), Australia, is shown in Fig. 8. The MV distribution system is fed by a $132 / 11 \mathrm{kV}, 30$ MVA transformer equipped with an OLTC. There are 69 nodes for ULB-2 and 95 nodes for ULE-2. The total line length for ULB-2 is $26.4 \mathrm{~km}$ while for ULE-2 is $38.6 \mathrm{~km}$. There is one SVR which has been connected at node 33 of ULB-2 to maintain the secondary voltage while two SVRs are connected at node 19 and node 49 of ULE-2 in cascade with a synchronous machine based bio-diesel generator of $750 \mathrm{kVA}$ capacity at node 76 . The recorded total peak load of the ULB2 is 2.24 MVA and ULE-2 is 1.51 MVA. Both the feeders have tie connection by means of air break switches (ABS). The stipulated operating voltage limits for the test system are within $+/-10 \%$ from a nominal voltage.

The DG is modeled to operate in a power factor control mode for depicting conventional operation as well as voltage control mode for signifying the proposed operation. The distribution network is assumed to be operated as a balance three phase system. The line impedance for $11 \mathrm{kV}$ feeders is $(0.802+\mathrm{j} 0.365) \Omega / \mathrm{km}$. The type-B SVRs are modeled by incorporating 32 taps in the series winding. A representative demand pattern depicting the realistic network conditions is considered to test the effectiveness of the proposed on-line control strategy in managing the voltage regulation in the network.

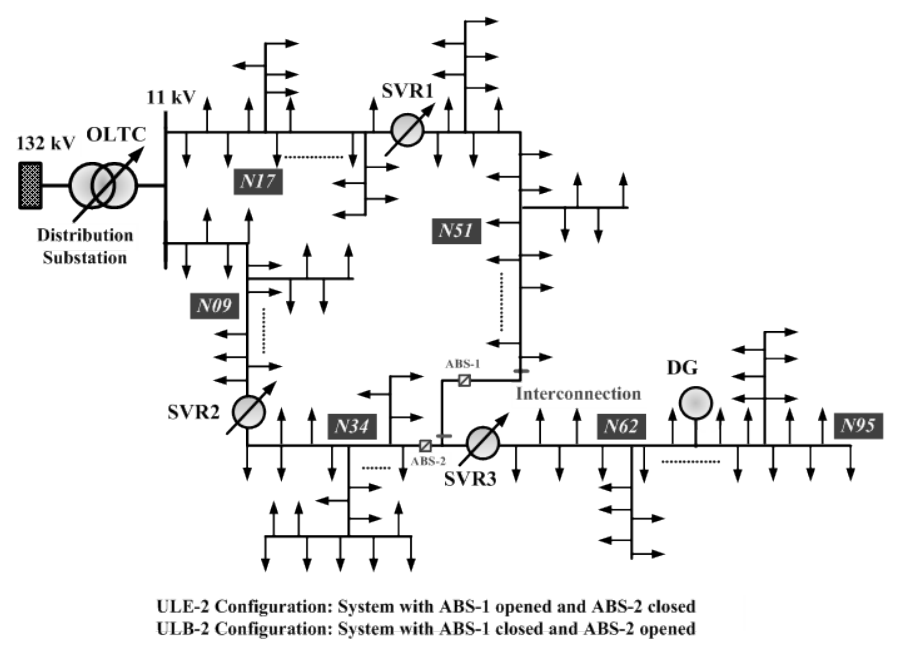

Fig. 8. Topology of test distribution system.

Table I and II depict the simulated demand patterns at different time intervals. The ULE2 feeder is lightly loaded compared to the ULB2 feeder.

TABLE I

SiMULATED DEMAND PATTERN FOR ULE-2

\begin{tabular}{c|c|c|c|c|c|c}
\multicolumn{7}{c}{ SIMULATED DEMAND PATTERN FOR ULE-2 } \\
\hline Time (s) & 0 & 100 & 200 & 300 & 500 & 600 \\
\hline Load (kVA) & 675 & 960 & 1140 & 1510 & 1270 & 995 \\
\hline
\end{tabular}

TABLE II

SIMULATED DEMAND PATTERN FOR ULB-2

\begin{tabular}{c|c|c|c|c|c|c}
\hline Time (s) & 0 & 100 & 200 & 300 & 500 & 600 \\
\hline Load (kVA) & 1234 & 1605 & 2082 & 2240 & 1890 & 1485 \\
\hline
\end{tabular}

It is assumed that the OLTC is operated with a fixed tap position. The initial tap positions for SVR1/SVR2 and SVR3 are arbitrarily assumed to be at 3 and 4 respectively in the direction of increasing voltage. The total simulated time delays for SVR1/SVR2 and SVR3 operation are assumed to be $4 \mathrm{~s}$ and $7 \mathrm{~s}$, respectively. The total mechanical time delay assigned for voltage regulating devices is $1.0 \mathrm{~s}$. Therefore, the total transition time for a tap operation is $1.0 \mathrm{~s}$. The time delays are modeled with constant time variant characteristics and nonsequential tap operation. The voltage reference values are set at $1.05 \mathrm{pu}$ and $1.01 \mathrm{pu}$ for OLTC and SVRs, respectively. The dead-band values are set at $+/-1.5 \%$ and $+/-2.0 \%$ for OLTC and SVRs, respectively. The simulated control parameters of proposed DG voltage control module are $V_{\text {SET/P }}=0.98 \mathrm{pu}, a=$ $0.05 \mathrm{pu}, V_{t}=0.025 \mathrm{pu}, \varepsilon_{t}=0$, and $T_{D G}=1.0 \mathrm{~s}$, where nominal value of DG excitation reference voltage is $0.90 \mathrm{pu}$ and pilot bus is the remote end bus of ULE-2 (N95). Based on the generator capability curve, the governor is tuned to supply an active power of $600 \mathrm{~kW}$ while the reactive power varies between $+450 \mathrm{kVAr}$ to $-350 \mathrm{kVAr}$.

\section{B. Simulation Results and Discussion}

The proposed control modules are modeled using MATLAB. The on-line information is assigned by 'Goto' and 'From' signal blocks without propagation delay. The total 
simulation time is assumed to be $750 \mathrm{~s}$. The simulation results for the independent operation of the ULE-2 feeder (by opening ABS-1 and closing ABS-2 in Fig. 8) are shown below. Fig. 9 shows the tap operations of multiple voltage regulating devices with conventional voltage control while Fig. 10 shows the tap operations with the proposed voltage control. Figs. 10 (b) to (e) are the zoomed versions of Fig. 10 (a) to clearly observe the tap operations.

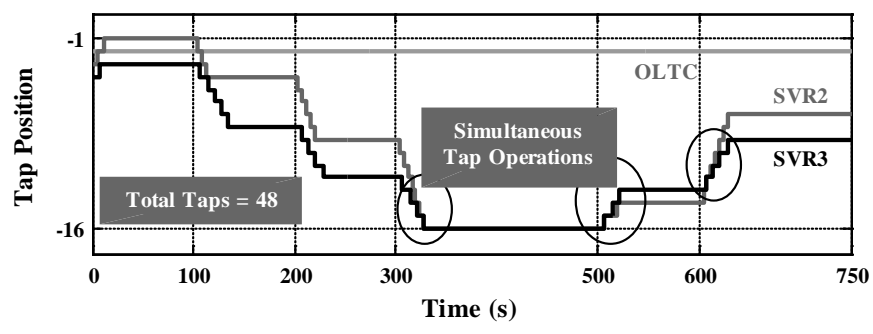

Fig. 9. Tap operations for ULE2 operation with conventional voltage control.

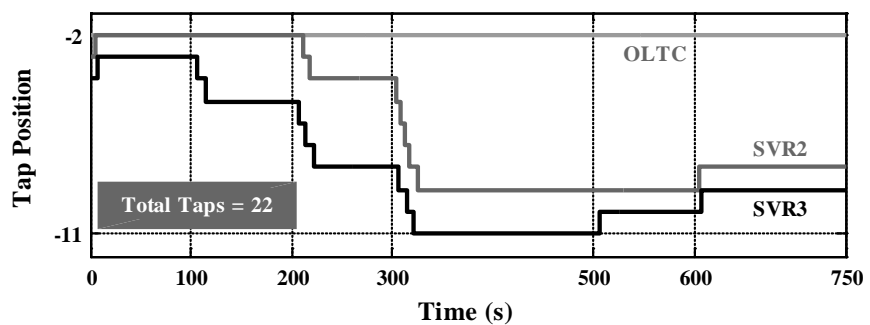

(a)

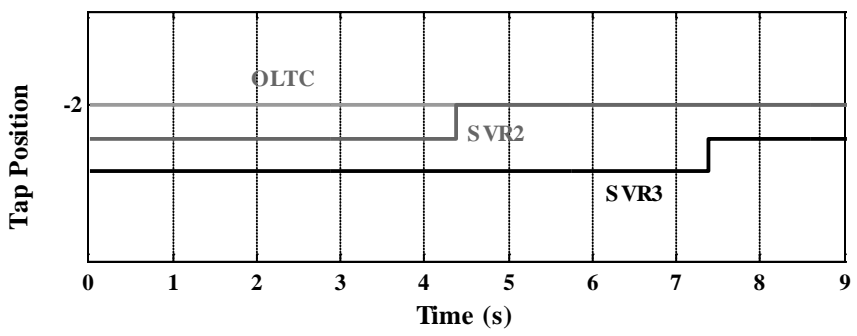

(b)

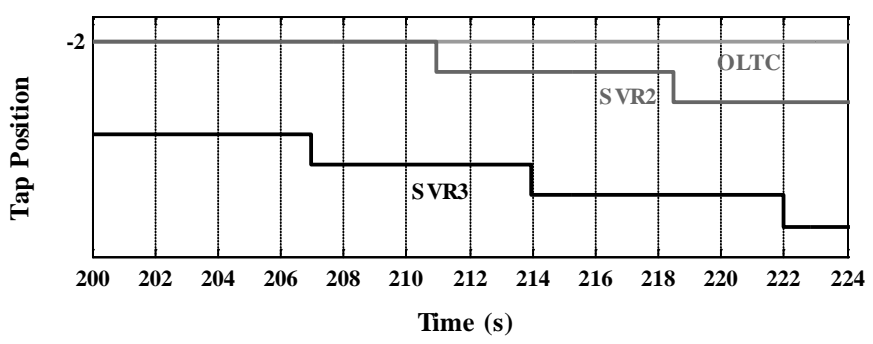

(c)

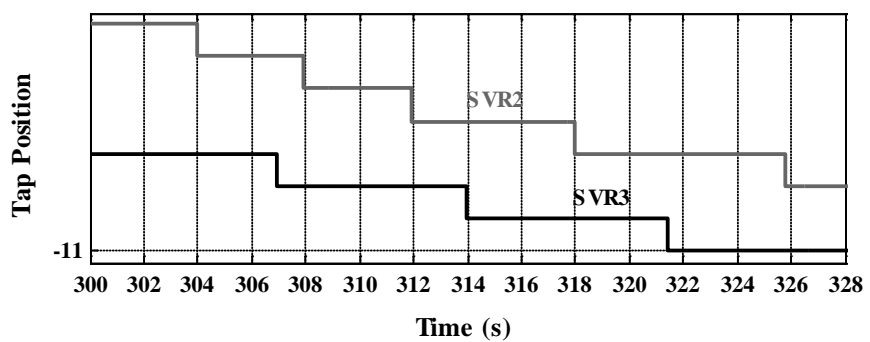

(d)

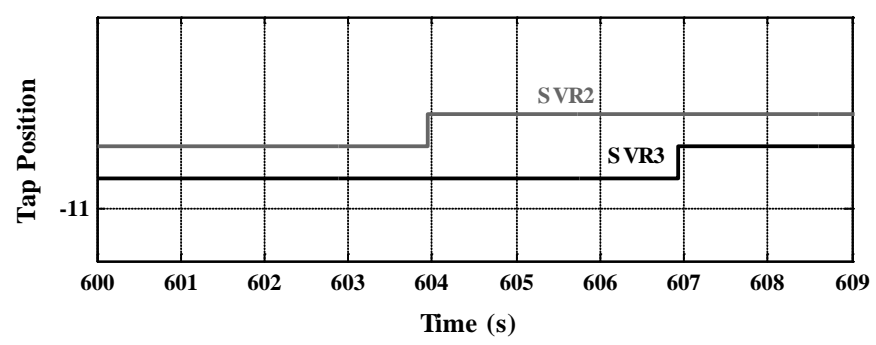

(e)

Fig. 10. Tap operations for ULE2 operation with proposed voltage control (a) over the total simulation period (b) from $t=0$ to $9 \mathrm{~s}$ (c) from $\mathrm{t}=200 \mathrm{~s}$ to 224 $\mathrm{s}(\mathrm{d})$ from $\mathrm{t}=300 \mathrm{~s}$ to $328 \mathrm{~s}$ (e) from $\mathrm{t}=600 \mathrm{~s}$ to $609 \mathrm{~s}$.

Fig. 9 shows simultaneous tap operations for SVRs at $\mathrm{t}=$ $328 \mathrm{~s}, 507 \mathrm{~s}$, and $628 \mathrm{~s}$ with conventional voltage control. According to Figs. 10 (a) to (e), there are no simultaneous tap operations (and DG-VCM actions) with the proposed control. It is because of the proposed CM-BSO based control action, which blocks the simultaneous operations in real-time. Its integrated operation with the modified local controllers also prioritizes the operation of DG-VCM and SVRs. The DGVCM and SVRs operate in accordance with the Step - 2 (vii) of the control algorithm as indicated in $\operatorname{IV}(C)$. The DG-VCM operates at $\mathrm{t}=1.0 \mathrm{~s}$ and $V_{\text {ref }}$ is updated from $0.90 \mathrm{pu}$ to $0.95 \mathrm{pu}$ based on the pilot bus voltage (N95). It also operates at $\mathrm{t}=2.0$ $\mathrm{s}$ and $V_{\text {ref }}$ is updated from 0.95 pu to 1.00 pu based on the pilot bus voltage. According to the DG-VCM control logic as given in (8) to (12), DG-VCM operates at $\mathrm{t}=301 \mathrm{~s}$. However, $V_{\text {ref }}$ doesn't change due to the constrained operation of DG within the associated reactive power capability limits. The postupdated DG excitation controller regulates the voltage with its fast control action. This DG-VCM based operation may also support the feeder voltage regulation at the upstream nodes.

The first tap operation of SVR2 (at $\mathrm{t}=4 \mathrm{~s}$ ) and SVR3 (at $\mathrm{t}$ $=7 \mathrm{~s}$ ) are initiated after DG-VCM control action followed by the time delay sequence given by (6). The $2^{\text {nd }}$ (at $t=108 \mathrm{~s}$ ) and $3^{\text {rd }}$ (at $t=115 \mathrm{~s}$ ) tap operations of SVR3 are initiated as given by Step - 2 (iii) of the control algorithm where there are no DG-VCM control action and/or SVR2 tap operation. Similarly, in response to the voltage drop at $\mathrm{t}=200 \mathrm{~s}$ and $\mathrm{t}=$ 300 s, SVR2 and SVR3 taps operate as given by Step - 2 (vi) of the control algorithm. Also, a SVR3 tap operates as given by Step - 2 (iii) in response to voltage change at $\mathrm{t}=500 \mathrm{~s}$, and SVR2 and SVR3 operate one tap each as given by Step -2 (vi) at $\mathrm{t}=600 \mathrm{~s}$.

As the result of maximized DG voltage regulation support by DG-VCM and the real time operation of CM-BSO, it can be seen that there is a significant reduction in tap operations for feeder ULE-2 operation with the proposed voltage control. This significant reduction in tap operations will obviously be beneficial to the distribution network operator, as it directly correlates to the decrement in the regulator maintenance cost, increase in its life time expectancy, and the decrease in intermittent voltage variations attributed to the tap operations.

The effect of proposed voltage control strategy to regulate the voltage of ULE-2 feeder can be observed in the selected 
nodes, which are N95 (at the end of the ULE-2 feeder), N09 (between substation and SVR2), N34 (between SVR2 and SVR3), and N62 (between SVR3 and DG) as shown in Fig. 8. The respective nodal voltage profiles for feeder operation with conventional voltage control and proposed voltage control are shown in Figs. 11 to 13. The reduction in resulting voltage variations attributed to the tap operations is clearly observed in these figures.

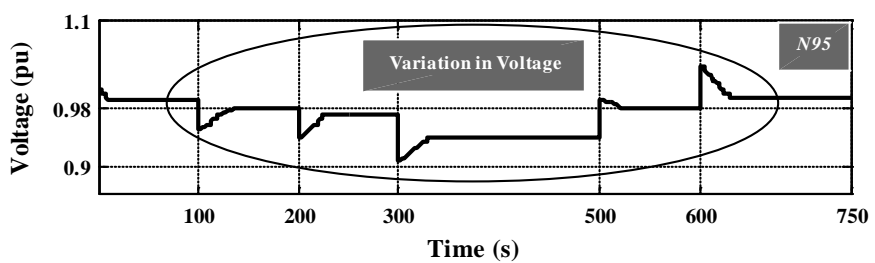

(a)

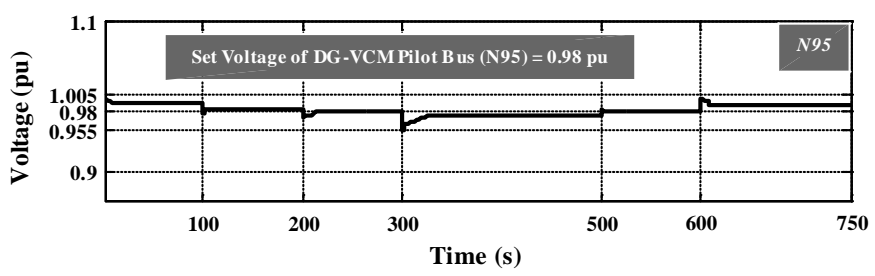

(b)

Fig. 11. Voltage at node N95 for ULE-2 operation with (a) conventional voltage control (b) proposed voltage control.

According to Figs. 11 (a) and (b), it can be seen that the DG-VCM regulates the pilot bus voltage (which is the remote end of the feeder, N95) within its stipulated limits of $1.005 \mathrm{pu}$ and $0.955 \mathrm{pu}$ for a set value of $0.98 \mathrm{pu}$, as outlined in the control logic given in (8) to (12). As shown in Fig. 11 (b), the voltage regulation at the end of the ULE-2 feeder is quite significant with the proposed control. Consequently, the proposed DG-VCM can support the feeder voltage regulation at each node in accordance with (4).

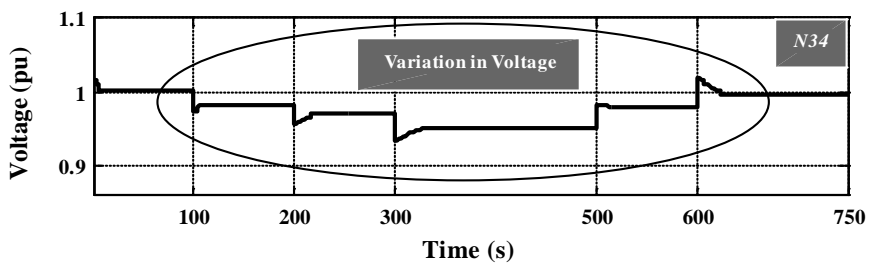

(a)

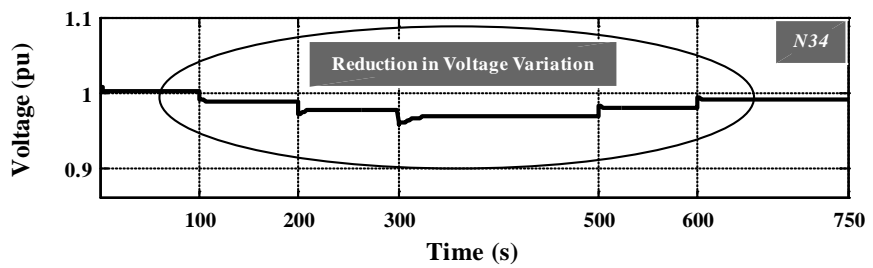

(b)

Fig. 12. Voltage at node N09 for ULE-2 operation with (a) conventional voltage control (b) proposed voltage control.

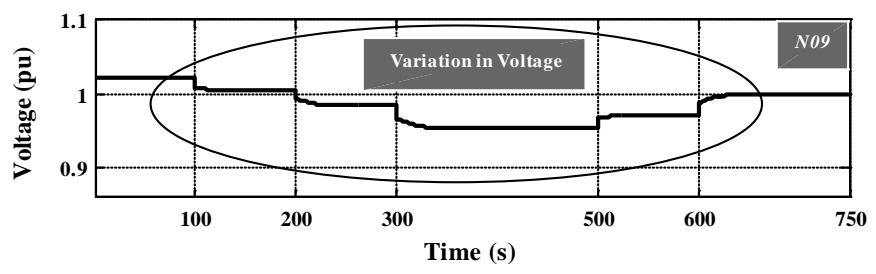

(a)

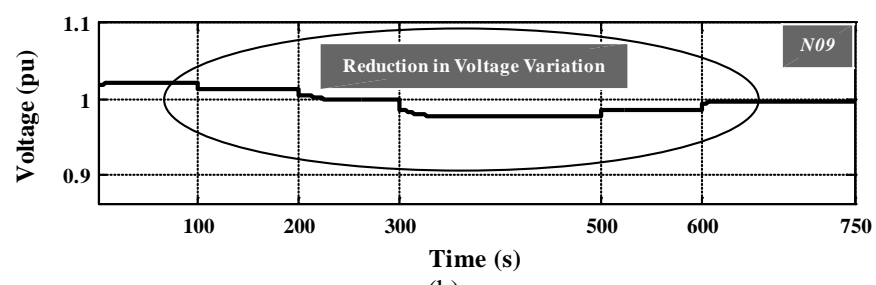

(b)

Fig. 13. Voltage at node N34 for ULE-2 operation with (a) conventional voltage control (b) proposed voltage control.

As seen in Figs. 12 and 13, the voltages at various nodes along the feeder are significantly regulated even under reduced tap operations of the SVRs. The voltage variations also reduce with the adoption of proposed voltage control strategy. Moreover, the overall voltage profile of the feeder improvises. It is observed that the similar results can be obtained for node N62. The active and reactive power outputs of the DG for ULE-2 operation are shown in Fig. 14.

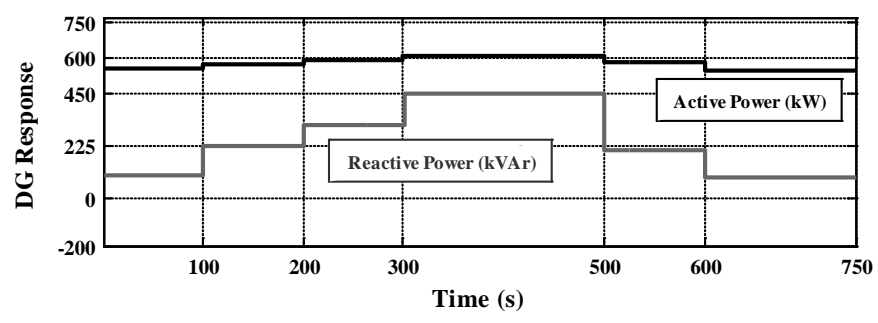

Fig. 14. Active and reactive power outputs of DG in ULE-2 configuration.

The simulation results for the interconnected operation of ULB-2 feeder involving a feeder section of ULE-2 (by closing ABS-1 and opening ABS-2 in Fig. 8) are shown below. Fig. 15 shows the tap operations of multiple voltage regulating devices with conventional voltage control. Figs. 16 (a) to (d) show the tap operations with proposed voltage control.

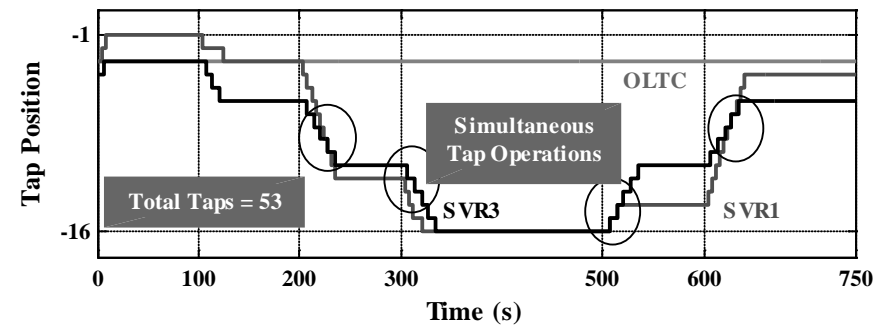

Fig. 15. Tap operations for ULB-2 interconnected operation with conventional voltage control. 


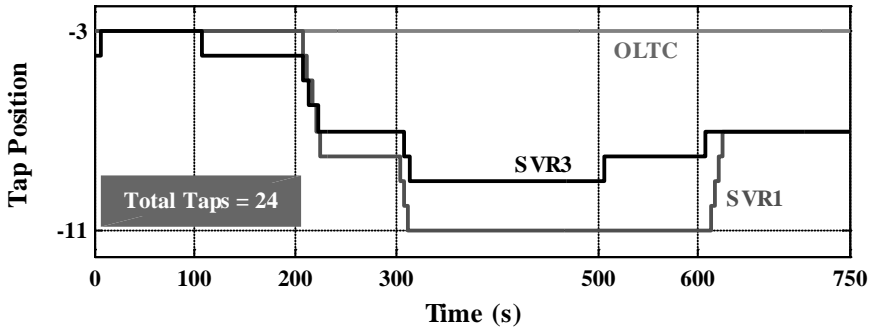

(a)

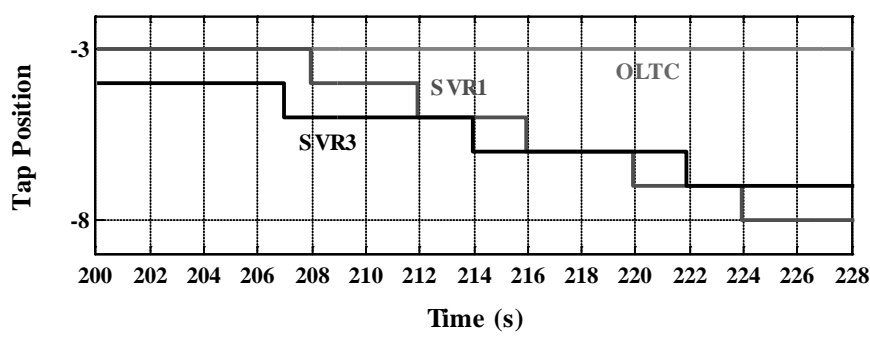

(b)

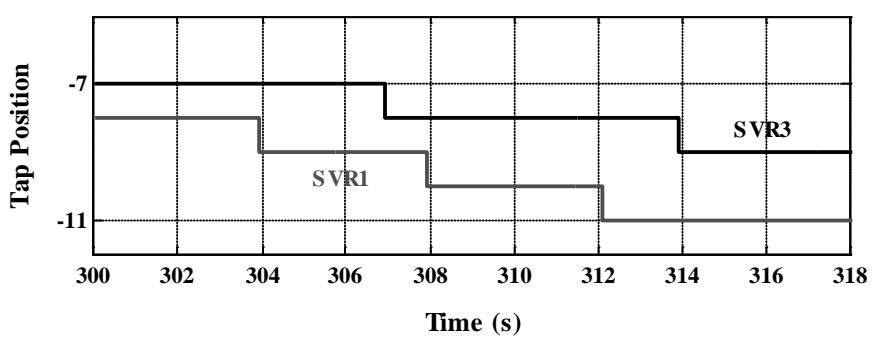

(c)

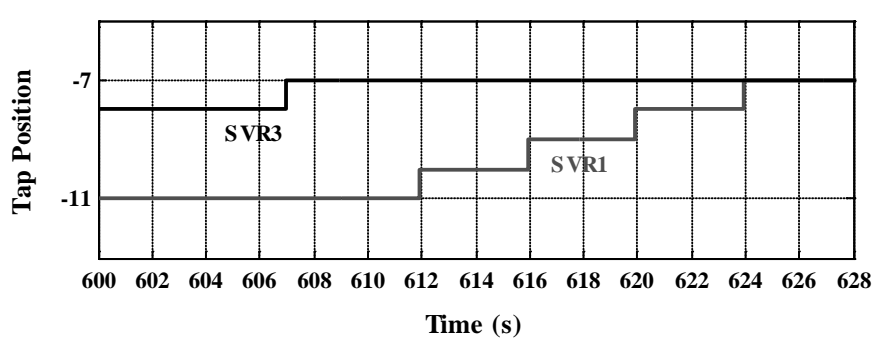

(d)

Fig. 16. Tap operations for ULB2 interconnected operation with proposed voltage control (a) over the total simulation period (b) from $t=200 \mathrm{~s}$ to $228 \mathrm{~s}$ (c) from t $=300 \mathrm{~s}$ to $318 \mathrm{~s}(\mathrm{~d})$ from $\mathrm{t}=600 \mathrm{~s}$ to $628 \mathrm{~s}$.

Fig. 15 shows simultaneous tap operations for SVRs at $\mathrm{t}=$ 228 s, 236 s, 321 s, 507 s, 514 s and 628 s with conventional voltage control. Figs. 16 (b) to (d) are the zoomed versions of Fig. 16 (a) to clearly observe the tap operations with the proposed voltage control. It is shown in Figs. 16 (a) to (d) that there are no simultaneous tap operations (and DG-VCM actions) with the proposed control. The DG-VCM operates at (i) $\mathrm{t}=1.0 \mathrm{~s}$ updating $V_{\text {ref }}$ from $0.90 \mathrm{pu}$ to $0.95 \mathrm{pu}$, (ii) at $\mathrm{t}=2.0$ $\mathrm{s}$ updating $V_{\text {ref }}$ from $0.95 \mathrm{pu}$ to $1.00 \mathrm{pu}$, and (iii) at $\mathrm{t}=601 \mathrm{~s}$ updating $V_{\text {ref }}$ from 1.00 pu to 0.95 pu based on the pilot bus voltage. The effect of proposed voltage control strategy to regulate the voltage of an interconnected ULB2 feeder can also be observed in the selected nodes, which are N95, N51 and N17 as shown in Figs. 17, 18 and 19 respectively. The results are very much similar to the previously simulated case of ULE2. The active and reactive power outputs of the DG for ULB-2 operation are shown in Fig. 20.

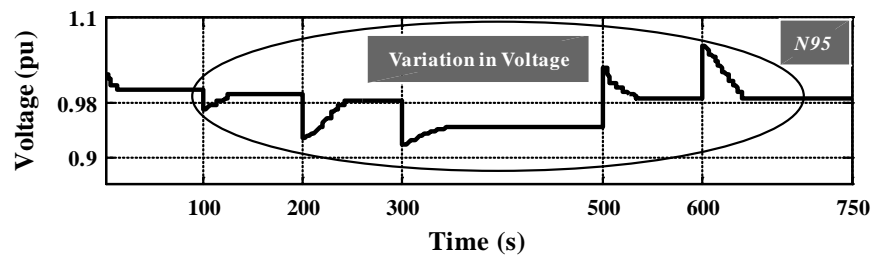

(a)

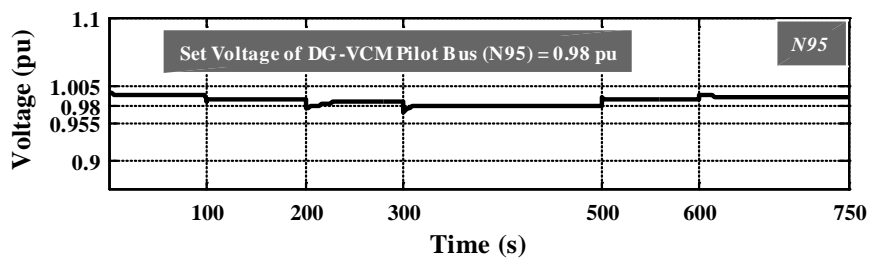

(b)

Fig. 17. Voltage at node N95 for ULB2 interconnected operation with (a) conventional voltage control (b) proposed voltage control.

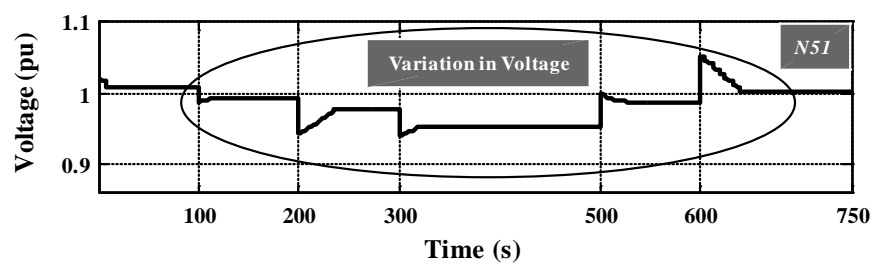

(a)

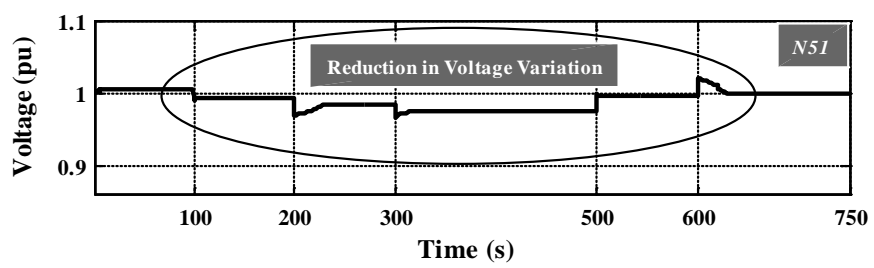

(b)

Fig. 18. Voltage at node N51 for ULB2 interconnected operation with (a) conventional voltage control (b) proposed voltage control.

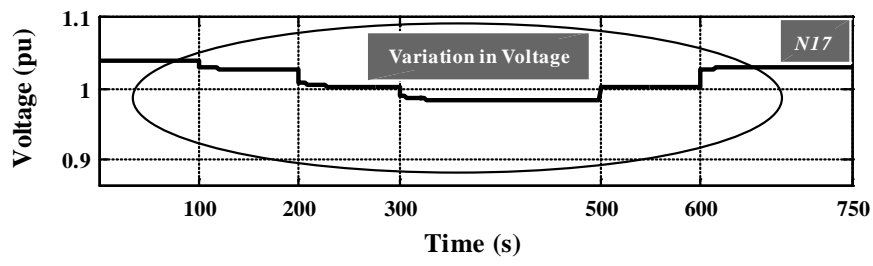

(a)



(b)

Fig. 19. Voltage at node N17 for ULB2 interconnected operation with (a) conventional voltage control (b) proposed voltage control. 


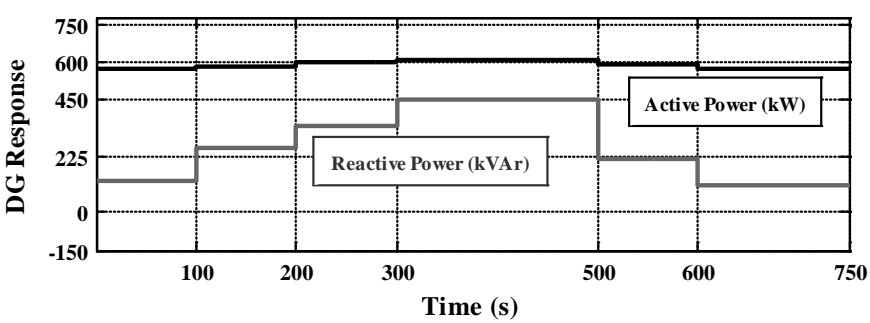

Fig. 20. Active and reactive power outputs of DG in ULB-2 configuration.

Further, simulation results for the interconnected ULB-2 feeder are also indicative of the fact that the performance of proposed on-line voltage control supersedes the performance of the conventional voltage control strategy. Based on the simulation studies, it can be concluded that the proposed DGVCM, CM-BSO based algorithm and its real-time implementation via substation centered DMS can effectively improve the steady state voltage profile of the distribution feeder containing SVRs and DG.

\section{CONCLUSION}

In this paper, a real-time on-line voltage control strategy is proposed for voltage regulation of distribution system with multiple regulating devices and DG. The control design is based on three main aspects: (a) maximizing voltage regulation support by DG through DG-VCM (b) procuring on-line load center voltage measurements for SVRs, and (c) real-time control for avoiding simultaneous operations of multiple voltage regulating devices and DG-VCM by means of CMBSO. Based on the time domain simulation studies conducted for realistic feeder operations, it can be seen that the proposed control strategy minimizes the total tap operations of SVRs while achieving voltage regulation support by regulating devices and DG. It also exploits the reactive capability of the DG unit in order to maximize the voltage regulation support. The proposed method reduces the resulting voltage variations attributed to the tap operations and improvises the feeder voltage. The simulation results have revealed that effective online voltage control for a distribution feeder can be achieved in real-time through the application of the proposed voltage control strategy.

\section{ACKNOWLEDGMENT}

The authors gratefully acknowledge the support by the personnel at Endeavour Energy in providing data and relevant information for the distribution system test feeders.

\section{REFERENCES}

[1] F. A. Viawan, and D. Karlsson, "Voltage and Reactive Power Control in Systems with Synchronous Machine-Based Distributed Generation," IEEE Trans. Power Delivery, vol. 23, pp. 1079-1087, Apr. 2008.

[2] F. A. Viawan and D. Karlsson, "Voltage and Reactive Power Control in Closed Loop Feeders with Distributed Generation,” in Proc. 2007 IEEE Power Tech Russia Conf., pp. 549-554.

[3] L. A. Kojovic, "Modern Techniques to Study Voltage Regulator-DG Interactions in Distribution Systems", in Proc. 2008 IEEE/PES Transmission and Distribution Conf., pp. 1-6.
[4] F. Bignucolo, R. Caldon, and V. Prandoni, "Radial MV Networks Voltage Regulation with Distribution Management System Coordinated Controller," Electric Power Systems Research, vol. 78, pp. 634-645, Apr. 2008.

[5] Dothinka Ranamuka, A. P. Agalgaonkar, and K. M. Muttaqi, "Investigating the Operation of Multiple Voltage Regulators and DG in a Distribution Feeder,” Energy Procedia, vol. 14, pp. 1945-1950, Mar. 2012.

[6] J. Morren, S.W.H. de Haan and J.A. Ferreira, "Contribution of DG units to voltage control: Active and reactive power limitations," in Proc. 2005 IEEE Power Tech Russia Conf., pp. 1-7.

[7] A. D. T. Le, M. A. Kashem, M. Negnevitsky and G. Ledwich, "Control Strategy of Distributed Generation for Voltage Support in Distribution Systems," in Proc. 2006 IEEE Power Electronics, Drives and Energy Systems International Conf., pp. 1-6.

[8] P. N. Vovos, A. E. Kiprakis, A. R. Wallace, and G. P. Harrision, "Centralized and Distributed Voltage Control: Impact on Distributed Generation Penetration,” IEEE Trans. on Power Systems, vol. 22, pp. 476-483, Feb. 2007.

[9] A. D. T. Le, M. A. Kashem, M. Negnevitsky and G. Ledwich, "Distributed Generation Control using Protection Principles," in Proc. 2006 AUPEC Universities Power Engineering Conf., pp. 1-6.

[10] T. Tran-quoc, E. Monnot, G. Rami, A. Almeida, C. Kieny and N. Hadjsaid, "Intelligent Voltage Control in Distribution Network with Distributed Generation,” in Proc. $200719^{\text {th }}$ Electricity Distribution International Conf., pp. 1-4.

[11] A. Darabi, S. A. Soleamani, and A. Hassannia, "Fuzzy Based Digital Automatic Voltage Regulator of a Synchronous Generator with Unbalanced Loads," American Journal of Engineering and Applied Sciences, vol. 1, pp. 280-286, 2008.

[12] O. Richardot, A. Viciu, Y. Besanger, N. Hadjsaid and C. Kieny, "Coordinated Voltage Control in Distribution Networks Using Distributed Generation,” in Proc. 2006 IEEE/PES Transmission and Distribution Conf., pp. 1196-1201.

[13] A. R. D. Fazio, G. Fusco and M. Russo, "Performance Comparison of Different Voltage Control Schemes on Distributed Generation," in Proc. 2006 IEEE Power Electronics, Drives and Energy Systems International Conf., pp. 1-6.

[14] W. H. Kersting, Distribution System Modeling and Analysis, vol. 1. Boca Raton: CRS Press, 2002, p. 204.

[15] J. J. Grainger, and S. Civanler, "Volt/Var Control on Distribution Systems with Lateral Branches Using Shunt Capacitors and Voltage Regulators Part I: The Overall Problem,” IEEE Trans. Power Apparatus and Systems, vol. PAS-104, pp. 3278-3283, Nov. 1985.

[16] P. Kundur, Power System Stability and Control, vol. 1. New York: McGraw-Hill, 1994, p. 683-687.

[17] NR Electric Corporation. Distribution Management System. [Online]. Available: http://www.nrelect.com

[18] B. Uluski, "Distribution Management Systems," presented at the CRN Summit, Cleveland Ohio, 2011.

[19] ABB Pvt. Ltd. Network Manger SCADA/DMS Distribution Network Management. [Online]. Available: http://www.abb.com

[20] S. Ghosal, and R. Mehrotra, "Detection of Composite Edges," IEEE Trans. Image Processing, vol. 3, pp. 14-25, Jan. 1994.

[21] J. H. Choi, and J. C. Kim,” Advanced Voltage Regulation Method of Power Distribution Systems Interconnected with Dispersed Storage and Generation Systems,” IEEE Trans. Power Delivery, vol. 16, pp. 329334, Apr. 2001. 